\title{
Gain, noise and intermodulation in a nonlinear superconducting resonator
}

\author{
Erik A Tholén ${ }^{1}$, Adem Ergül2, David Schaeffer ${ }^{2}$ and David B Haviland ${ }^{2 *}$
}

\author{
"Correspondence: haviland@kth.se \\ ${ }^{2}$ Nanostructure Physics, Royal \\ Institute of Technology (KTH), \\ Stockholm, Sweden \\ Full list of author information is \\ available at the end of the article
}

\begin{abstract}
A superconducting microwave resonator is modified with several weak links to make it nonlinear and operated as a phase-insensitive microwave amplifier. Signal gain is demonstrated by intermodulation with a strong pump. The gain is sharply frequency dependent, and we demonstrate phase dependence by examining correlations between the signal and one idler which is a 3rd order intermodulation product of the pump and signal tones. A calibration procedure is described which is based on measurement of both thermal and quantum noise, revealing that the following HEMT amplifier adds noise at 15 times the quantum limit. When operated as a phase-insensitive amplifier the nonlinear resonator added noise at 2.5 times the quantum limit. Significant power is found at intermodulation products beyond 3 rd order, which may be responsible for the inability to reach the quantum limit.
\end{abstract}

PACS Codes: 74.78.-w; 42.65.Yj; 85.25.Cp

Keywords: intermodulation; parametric amplifier; quantum noise

\section{Introduction}

Minimal dissipation and a designable nonlinearity are the important attributes of superconductors which are enabling the development of quantum electrodynamic circuits. These attributes are manifested in distributed microwave structures with eigenmodes that are suitably modified by tunnel junctions or weak links to make them nonlinear. Such circuits find use in: single photon detection [1], generating [2] and routing [3] single microwave photons, detecting the state of individual quantum bits [4], and even as the qubit itself [5]. An important general purpose application is microwave signal amplification at the quantum limit of added noise [6]. Here we present measurements of gain, noise and intermodulation in a superconducting nonlinear resonator operated as phase-insensitive signal amplifier. Good agreement is achieved between measurement and a theory that includes one parameter describing a cubic nonlinear term. However, we observe strong response at high-order intermodulation frequencies which is not included in the theory. We believe that power lost to high-order intermodulation is responsible for the inability of the amplifier to squeeze noise at the quantum limit.

\section{Amplification at the quantum limit}

A fundamental theorem of signal amplification states that any narrow-band linear amplifier providing significant power gain to a signal of arbitrary phase applied to its input, must add noise at its output, where the added noise power per unit bandwidth, or added

(C) 2014 Tholén et al.; licensee Springer on behalf of EPJ. This is an Open Access article distributed under the terms of the Creative Commons Attribution License (http://creativecommons.org/licenses/by/2.0), which permits unrestricted use, distribution, and reproduction in any medium, provided the original work is properly cited. 
noise energy, is at least one half the zero-point fluctuation energy at the signal frequency [7].

$$
E_{N}^{\text {added }} \geq \frac{1}{2} h f_{s}
$$

It is however possible to create amplifiers that are not subject to this minimum added noise theorem. The phase-sensitive parametric amplifier operates with a pump that periodically modulates a parameter of a linear system. Signals which drive the system at half the pump frequency are amplified when they are in phase with the pump, whereas quadrature signals are de-amplified. This squeezing of a signal results in overall power gain, where power is transferred from the pump to the signal, and it is in principle a reversible process that can be performed with no added noise. Squeezing at the quantum limit produces a squeezed vacuum state, as recently demonstrated [8].

The utility of a phase-sensitive amplifier is however limited to applications where one has prior knowledge of the phase of the input signal, which is often not the case. A phaseinsensitive amplifier is more useful as a general purpose measurement tool and there is growing interest in phase-insensitive amplification with noise performance at the quantum limit [9-15]. In its simplest form, phase-insensitive amplification is realized when a nonlinear system mixes two drive tones, a strong pump tone at frequency $f_{p}$ and a much weaker signal tone at frequency $f_{s}$, to generate an idler tone at $f_{i}=f_{p}-f_{s}$. This mixing can provide gain for arbitrary phase of the signal and squeezing becomes apparent in the correlation between the externally applied signal tone and the internally generated idler tone, which are both locked to the same pump. This type of squeezing is also in principle a reversible amplification of the signal which can be undone if both the signal and idler are applied to another identical nonlinear resonator with an appropriately phase-shifted pump. The reversibility of the process implies that it can be performed without added noise.

The idler in this most simple case is generated by intermodulation of the applied signal and pump tones and it can be understood as resulting from a quadratic nonlinearity. Consider a standing wave in a transmission line with a current-dependent inductance $L(i)$. The flux $\Phi(t)$, which is defined in terms of the potential at one end of the line, $V=\frac{d \Phi}{d t}$, obeys a nonlinear equation of motion.

$$
\frac{1}{\left(2 \pi f_{0}\right)^{2}} \frac{d^{2} \Phi}{d t^{2}}+\frac{1}{2 \pi f_{0} Q} \frac{d \Phi}{d t}+\Phi+\sum_{n=2}^{N} g_{n} \Phi^{n}=\Phi_{\text {drive }}(t)
$$

The coefficients $g_{n}$ describe an arbitrary conservative nonlinearity of degree $N$ and $f_{0}$ and $Q$ are the characteristic frequency and quality factor of the linear resonator. To first order in the nonlinear perturbation, a drive consisting of two tones will generate a correction to the linear response of the resonator containing terms of the form,

$$
\sim g_{n}\left[A_{s} e^{i 2 \pi f_{s} t}+A_{p} e^{i 2 \pi f_{p} t}\right]^{n}
$$

Expanding this binomial, we see that the quadratic nonlinearity generates terms $\sim g_{2} \times$ $e^{i 2 \pi\left(f_{p} \pm f_{s}\right) t}$ which contains the idler discussed above. 
Generally, to any order in a perturbative expansion (so long as it converges) and for a nonlinearity of arbitrary degree, response to a two-tone drive is generated only at frequencies which are intermodulation products of the drive tones,

$$
f_{\mathrm{IMP}}=n_{s} f_{s}+n_{p} f_{p}
$$

where $n_{s}$ and $n_{p}$ are integers. The order of the intermodulation product is given by $\left|n_{s}\right|+\left|n_{p}\right|$. In nonlinear optics second-order intermodulation is referred to as three-wave mixing, describing the interaction of three electromagnetic waves travelling through a medium having a $\chi_{2}$ or quadratic nonlinearity. This type of nonlinearity occurs in crystals which lack a point of inversion symmetry [16]. For superconducting resonators the Josephson junction or weak link nonlinearity has a current-flux relation which is an odd function, $i(+\Phi)=-i(-\Phi)$, leading to a nonlinear oscillator equation with only odd powers of $\Phi$, i.e. Eq. (2) with $g_{\text {even }}=0$. Therefore second order intermodulation is typically absent in passive Josephson devices. However, three-wave mixing or second order intermodulation can be realized in actively driven, or parametric Josephson devices, which are non-reciprocal in nature [17].

In the nonlinear resonator studied here we realized phase-insensitive amplification by third-order intermodulation of the signal and pump (4 wave mixing) where the idler is at frequency $f_{i}=2 f_{p}-f_{s}$. This intermodulation product can be generated by a nonlinear oscillator with a $g_{3}$ coefficient only, known as the Duffing oscillator or Kerr nonlinearity. A detailed theory of the cavity parametric amplifier perturbed by a Kerr nonlineary has been worked out by Yurke and Buks [9] and extended to the two port system measured here [18]. When comparing our measurements to this theory we could produce excellent agreement between the theory and experiment by adjusting the parameters of the theory $[19,20]$. Here we extend this analysis to measurements of noise. We also present measurements of higher order intermodulation products forming a frequency comb of response near resonance. These products are typically not looked for in experiments on parametric amplification, but we found significant power at these frequencies, especially when measuring de-amplification of a signal tone. We speculate that this higher order intermodulation is responsible for not being able to efficiently squeeze with our amplifier.

\section{Experimental details and calibration}

The nonlinear resonator is a thin-film $\mathrm{Nb}$ coplanar waveguide (CPW) fabricated with a photo lithography process by Star Cryoelectronics [21]. Input and output ports were coupled to the resonator by interdigitating the center line of the resonator with tapered CPW feed lines. We attempted to make asymmetric coupling with smaller interdigitating length at the input, but we found that both ports had nearly the same coupling, indicating the coupling is primarily due to fringing fields. The distributed resonator has a series of eigenmodes when its length is close to an integer multiples of the electromagnetic halfwavelength $L \simeq \frac{n \lambda}{2}$. Here we present measurements of the response in the vicinity of the fundamental eigenmode $(n=1)$ with $f_{0}=7.60845 \mathrm{GHz}$. A nonlinear inductance was created by milling out a series of 10 weak links with a focused ion beam, each approximately $30 \mathrm{~nm} \times 30 \mathrm{~nm}$ in cross section, placed close together in the middle of the resonator, where the oscillating suppercurrent has an anti-node. The sample is flip-chip mounted on to a PC board which transforms the coplanar geometry of the chip to coax input and output connectors. For further details and images see Ref. [20]. 


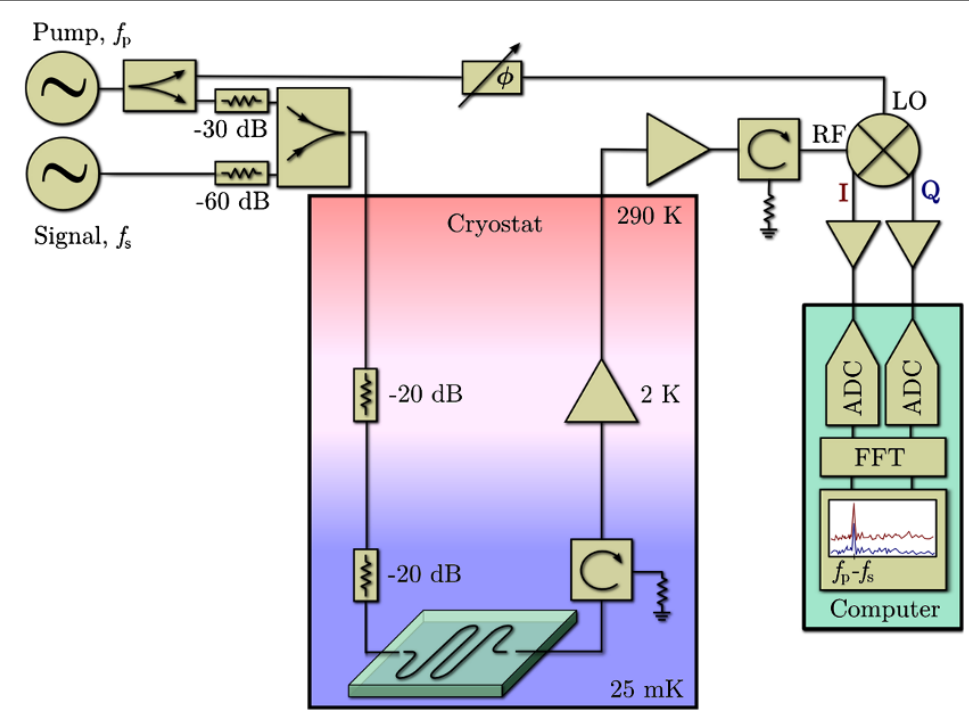

Figure 1 Schematic diagram of the microwave setup used to realize phase-insensitive amplification and homodyne detection.

A schematic diagram of the measurement setup is shown in Figure 1. The sample is mounted in a dilution refrigerator with base temperature $25 \mathrm{mK}$. The pump and signal tones are combined at room temperature and applied via a coax transmission line to the resonator input. Two stages of attenuation at low temperatures are used on the input side to bring the thermal radiation at the input into thermal equilibrium at the base temperature of the cryostat. Signals emerging from the output are sent through a circulator to a cryogenic pre-amplifier mounted at the $2 \mathrm{~K}$ stage of the cryostat. The circulator, with the third terminal connected to a $50 \Omega$ termination acts as an isolator, protecting the resonator from the back-action noise of the cryogenic preamplifier. The signal at the output of the preamplifier is amplified again by a room-temperature microwave amplifier followed by another isolator, and then an IQ mixer for homodyne detection using a phase-shifted copy of the pump signal as the local oscillator. In this arrangement, both the signal and idler tones will be coherently down-converted to the same frequency $\Delta f=f_{s}-f_{p}$. A variable delay shifts the phase of the pump signal, allowing one to probe the phase dependence of the signal and idler gains, that is the squeezing.

The cryogenic HEMT amplifier is the dominant source of noise in our setup. We tested amplifiers from Miteq, Amplitech, and Low Noise Factory [22], the later being far superior to the others with $\simeq 40 \mathrm{~dB}$ of gain in the band $3-8 \mathrm{GHz}$ with a DC power consumption of only $6 \mathrm{~mW}$. The back-action noise from at input stage of the HEMT amplifier is isolated from the resonator and brought in to thermal equilibrium at the base temperature of the cryostat by the $50 \Omega$ termination at one port of the cold circulator. If the $50 \Omega$ termination on the circulator is in equilibrium with the cryostat's calibrated restive thermometer, the noise emitted from this $50 \Omega$ source can used to calibrate the measurement system. Noise from this $50 \Omega$ source is measured in a very narrow frequency band (effective bandwidth $0.5 \mathrm{kHz}$ ) centred at the frequency $f_{c}$. The measurement band does not overlap with the resonance of the cavity so the noise is reflected at the output of the resonator, re-enters the circulator, and is transported to input of the HEMT amplifier. Thus the total noise power per unit bandwidth measured at the output of the mixer comprises the noise from 


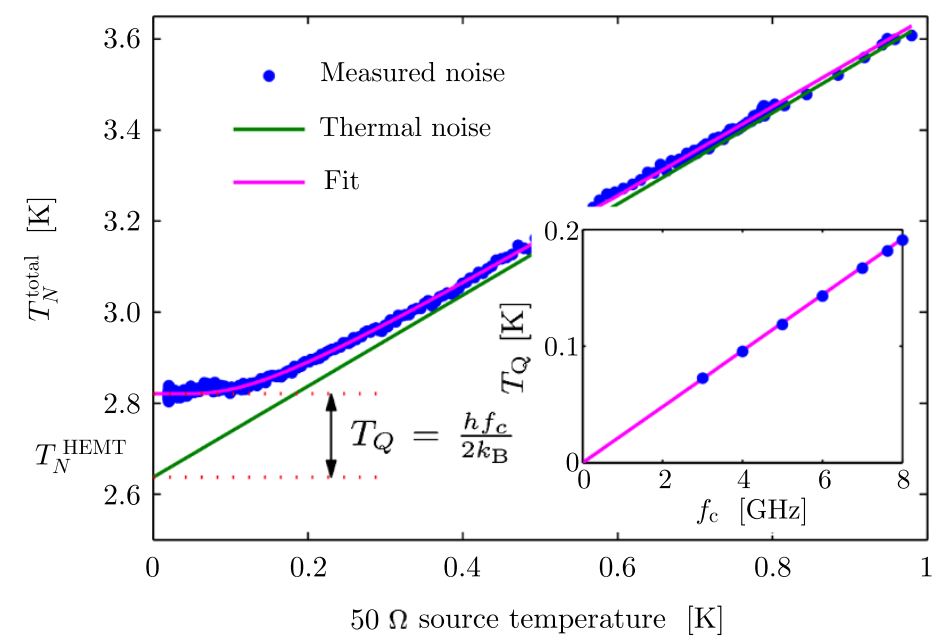

Figure 2 Microwave noise power is calibrated in a narrow band centered at the frequency $f_{c}=7.62 \mathrm{GHz}$, by varying the temperature of the $50 \Omega$ termination on the cold circulator.

A one-parameter fit to Eq. (5), adjusting only the overall gain factor, allows for determination of the HEMT amplifiers noise temperature $T_{N}^{\mathrm{HEMT}}=2.62 \mathrm{~K}$ and observation of the quantum noise $T_{Q}=0.18 \mathrm{~K}$. The data points in the inset are the quantum noise at different center frequencies in the band of the HEMT amplifier, and the straight line is the expected behavior $T_{Q}=\frac{h f_{C}}{2 k_{\mathrm{B}}}$.

this $50 \Omega$ source, plus the added noise of the HEMT amplifier.

$$
E_{N}^{\mathrm{total}}(T)=E_{N}^{\mathrm{HEMT}}+\frac{1}{2} h f_{c} \operatorname{coth}\left(\frac{h f_{c}}{2 k_{\mathrm{B}} T}\right)
$$

where we have used the quantum expression for the fluctuations from the $50 \Omega$ source. This simple expression assumes that all inputs and outputs are matched to $50 \Omega$, which is the case.

We measured the noise as a function of temperature and fit the theory Eq. (5) to the measured data as shown in Figure 2, where the noise is expressed as an effective temperature $T_{N}=\frac{E_{N}}{k_{\mathrm{B}}}$. The least-squared fit was obtained by adjusting only one parameter, the overall scale factor on the vertical axis. This scale factor contains the gains and attenuations from all of the various components in our measurement chain, which would not be easy to calibrate independently. At high temperatures $k_{\mathrm{B}} T>h f_{c}$ the data approach the Johnson-Nyquist thermal noise from the $50 \Omega$ source, which the fit determines as the straight green line in Figure 2. The $T=0$ intersection of this line with the noise axis gives the added noise of the HEMT amplifier, $T_{N}^{\mathrm{HEMT}}=2.62 \mathrm{~K}$. The difference between the amplifiers added noise and the measured noise extrapolated to $T=0$, is the quantum noise, $T_{Q}$. We performed this measurement at several center frequencies $f_{c}$ to verify that the quantum noise determined by this fitting procedure behaved as expected $T_{Q}=\frac{h f_{c}}{2 k_{\mathrm{B}}}$ ( inset of Figure 2).

Thus it is possible to use noise to calibrate the microwave measurement chain against the cryostat's calibrated restive thermometer. We are also able to directly measure the quantum noise with the HEMT amplifier, which adds only $T_{N}^{\mathrm{HEMT}} \simeq 15 T_{Q}$ to the total noise. In contrast to two-point calibrations involving low temperature switches [23], or exotic hot quasiparticale noise sources [12], this straight-forward calibration method is rather simple to perform. Our confidence in the accuracy of this method was bolstered 


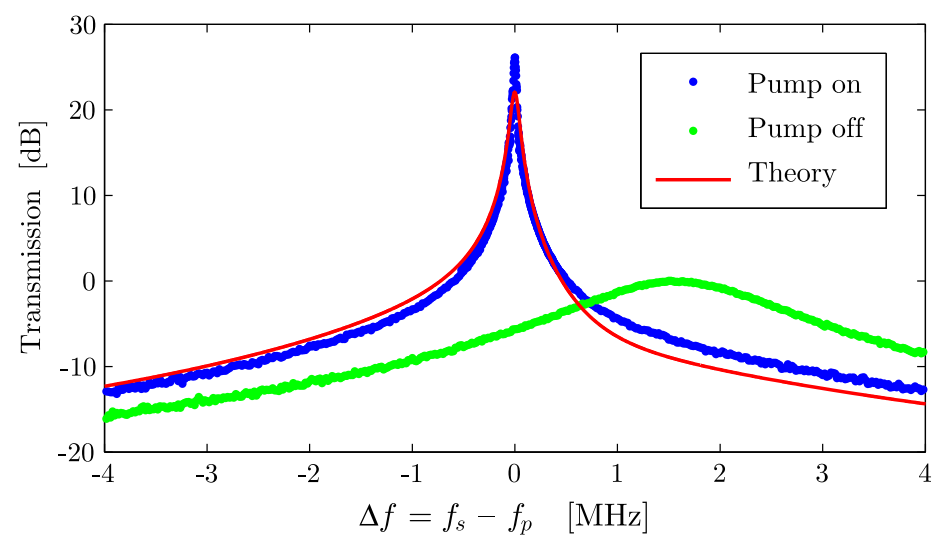

Figure 3 Frequency response of the parametric amplifier with the pump turned off and the pump turned on. The pump frequency is $f_{p}=7.6069 \mathrm{GHz}$ which is $1.55 \mathrm{MHz}$ below the resonant frequency of the resonator in the linear regime. The pump power is $-83.8 \mathrm{dBm}$ at the input of the sample box. The red line is a fit to the theory made by adjusting the strength of the Kerr nonlinearity.

when we observed that problems with the $50 \Omega$ source not being in thermal equilibrium with the cryostats thermometer were clearly evident in the poor quality of the fit to Eq. (5) to the data [20]. This calibration method has been use for Planck spectroscopy of thermal microwave states [24].

\section{Measurements and analysis}

Signal amplification by the nonlinear resonator is demonstrated in Figure 3 which shows a measurement of the signal transmission when the pump power and frequency are fixed at $P_{p}=-83.8 \mathrm{dBm}$ and $f_{p}=7.6069 \mathrm{GHz}$, very close to bifurcation point of the nonlinear resonator. In this measurement the signal source and mixer are replaced by the output and input ports of a network analyser respectively, and the signal power is fixed to $P_{s}=$ $-133.7 \mathrm{dBm}$ while its frequency is swept. The measurement was calibrated so that $0 \mathrm{~dB}$ corresponds to unity transmission between the input and output connectors of the board on which the chip is mounted. The transmission curve at this signal power (green data in Figure 3 ) in the absence of the pump is also shown in Figure 3 which is well described by the Lorentzian lineshape of a driven, damped harmonic oscillator with resonant frequency $f_{0}=7.60845 \mathrm{GHz}$ and width $\gamma=900 \mathrm{kHz}\left(Q=\frac{2 \pi f_{0}}{\gamma}=8,454\right)$.

When the pump is turned on we observe gain in a rather narrow band which is sharply peaked at zero detuning between the pump and signal, with a maximum gain of $28 \mathrm{~dB}$ (blue data points in Figure 3). Using the values of $f_{0}$ and $Q$ determined from the transmission curve in the linear regime, and the value of a cubic nonlinear term determined by fitting transmission curves at many different signal powers, when the resonator is in the nonlinear regime (data not shown) we could calculate the theoretically expected frequency dependent gain $[9,18]$ (red line in Figure 3). Good correspondence with the measured signal gain is seen over many times the resonator bandwidth. We found that including the nonlinear damping parameter in the theory did not improve the quality of the fit.

In a previous publication [19] we reported a detailed study of the pump power and phase dependence of the signal and idler gain, where excellent agreement with theory $[9,18]$ was found. We were not able to achieve equal magnitude of the amplification and deamplification of a signal, which we understood as most likely being the result of too weak coupling 


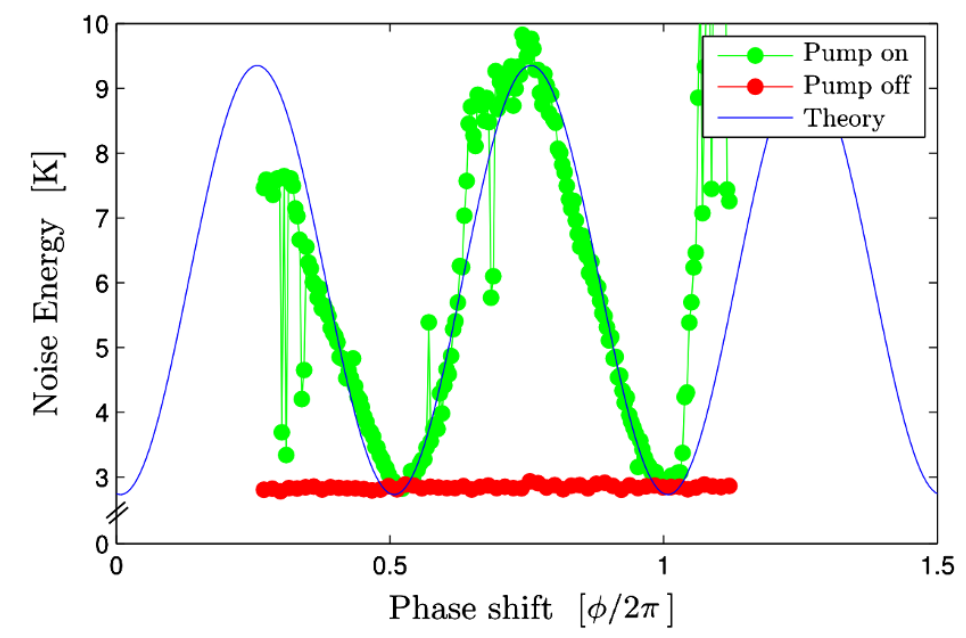

Figure 4 Phase dependence of the noise power in a $30 \mathrm{kHz}$ bandwidth, with the pump on (green) and pump off (red). The solid line is the theoretical expectation taking in to account the frequency dependence of the gain. Instabilities in the amplifier cause jumps in the measurements.

of the input and output ports. Here we report noise and intermodulation measurements which were made in a subsequent cool-down of the sample. The thermal cycling of the sample caused a slight shift of the bifurcation point and for the following measurements the working point of the pump was $f_{p}=7.6029 \mathrm{GHz}$ and $P_{p}=-84 \mathrm{dBm}$.

With the signal turned off we measured only the noise in a $30 \mathrm{kHz}$ bandwidth as the phase shifter is varied. Note that here we are measuring noise close to resonance, so the noise measured is inside the cavity and it is effected by the noise entering from both the input and output ports. In Figure 4 we see that noise at the output of the mixer is clearly phase-dependent, verifying that intermodulation with the pump is causing a correlation of the frequency components of a random signal that are equally spaced from pump, $f_{p} \pm \Delta f$. We see that when the phase shifter is adjusted for minimum noise (maximum de-amplification) we recover the independently measured value of the HEMT amplifiers added noise $2.6 \mathrm{~K}$. This value is the same as the noise measured when the pump is off (red data in Figure 4). When the phase shifter is adjusted for maximum gain we find that the nonlinear cavity amplifier adds and additional $6.8 \mathrm{~K}$ of noise to the total noise power. Taking in to account the frequency dependence and phase dependence of the gain, we can estimate the phase dependence of the total measured noise as,

$$
T_{N}^{\mathrm{total}}(\phi)=T_{N}^{\mathrm{HEMT}}+\int_{0}^{30 \mathrm{kHz}} G(\phi, f)\left(T_{Q}+T_{N}^{\mathrm{NLO}}\right) d f
$$

where $T_{N}^{\mathrm{NLO}}$ is the temperature of the added noise of the nonlinear oscillator. This theoretical curve is shown in Figure 4 with $T_{N}^{\mathrm{NLO}}=0.44 \mathrm{~K}$. The jumps observed in the measured data are due to instabilities of the nonlinear oscillator which is biased close to the bifurcation point.

In another measurement we applied a signal with fixed frequency with $\Delta f=f_{s}-f_{p}=$ $10 \mathrm{kHz}$ and measured the power spectrum at the output of the mixer over a much wider frequency band corresponding to $\sim 10 \Delta f$. This measurement was made by Fast Fourier Transform (FFT) of the output of the mixer with a $1 \mathrm{kHz}$ resolution bandwidth, where 

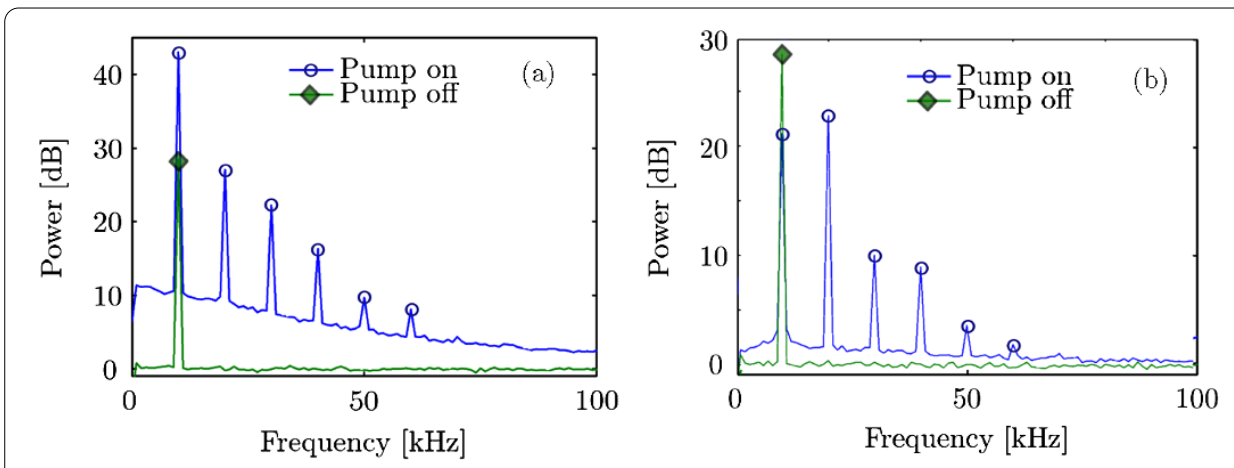

Figure 5 Power spectrum measured at the output of the mixer when a signal is applied at $\boldsymbol{\Delta} \boldsymbol{f}=\boldsymbol{f}_{\mathbf{s}}-\boldsymbol{f} \boldsymbol{p}=\mathbf{1 0} \mathrm{kHz}$. Measurements are shown when the pump is on (blue) and when the pump is off (green), for two cases: (a) the phase shifter is tuned for maximum amplification, and (b) the phase shifter is tuned for maximum de-amplification. The measurement reveals both signal and noise gain, and the additional peaks at integer multiples of $\Delta f$ are due to higher-order intermodulation products of the signal and pump.

1,000 power spectra were averaged. To avoid Fourier leakage between different components in the spectrum, we synchronized the microwave generators and the sampling clock on the ADC, and we chose both pump and signal frequencies to be integer multiples of the sampling frequency.

Figure 5 shows the averaged power spectra when the phase shifter is adjusted for maximum amplification (Figure 5(a)) and maximum de-amplification (Figure 5(b)). Measurements with the pump both on and off are shown in each figure. For these measurements, the power is normalized so that $0 \mathrm{~dB}$ corresponds to the pump off noise level. Comparing the height of the signal peak at $10 \mathrm{kHz}$ for the pump on and pump off case, we find $14 \mathrm{~dB}$ of amplification and $7 \mathrm{~dB}$ of de-amplification of the signal tone. Also evident is a large number of peaks at integer multiples of $\Delta f$, corresponding to higher order intermodulation products of the signal and pump. Between the peaks we can see the noise level, which reveals the frequency dependence of the amplifiers gain. In Figure 5(a) we find that the signal-to-noise ratio (SNR) at $10 \mathrm{kHz}$ improves by a factor of 5.9 when the pump is on. Since the pump off noise is measured to be that of the HEMT amplifier, we can determine the added noise of the nonlinear oscillator to be $T_{N}^{\mathrm{NLO}}=T_{N}^{\mathrm{HEMT}} / 5.9=0.44 \mathrm{~K}$, consistent with the previous measurement.

\section{Discussion and conclusions}

The peaks in Figure 5(a) and Figure 5(b) at integer multiples of $\Delta f$ correspond to odd order intermodulation products (e.g. $2 f_{p} \pm f_{s}, f_{p} \pm 2 f_{s}, 3 f_{p} \pm 2 f_{s}, 2 f_{p} \pm 3 f_{s}$ etc.). It is difficult to pin-point the source of these higher order intermodulation products: They may be generated in the nonlinear resonator as a result of expansion coefficients $g_{n}$ with odd $n>3$ in Eq. (3), or from the $g_{3}$ term in higher order perturbation theory. Nonlinearity of the mixer may also generate these higher order intermodulation products. In any case, participation of both signal and pump is required, and power is taken from the signal band in their creation. The amplitude of the higher order intermodulation products is quite large, with the 5th order product exceeding the signal amplitude at maximum de-amplification and intermodulation up 15th order can be resolved above the noise. These higher order products are clearly diminishing our ability to measure gain and deamplification of the 
signal, and we speculate that they also inhibiting the measurement of noise squeezing at the quantum limit.

One way in which one might control this loss of power to higher order intermodulation is to filter the higher-order IMPs by designing a system where the linear response is negligible at undesirable intermodulation products. Another strategy would be to design a drive consisting of a frequency comb with the amplitude and phase of each component appropriately tuned to cancel the undesired response. In fact, these IMPs can be measured in a phase-coherent way and used for accurate reconstruction of the actual nonlinearity [25]. Development is under way to make system which will allow for driving and measuring response with arbitrary, phase-coherent frequency combs [26].

In this paper we measured intermodulation response near one resonance and analyzed the data within the context of a basic theory of a nonlinear system with only two degrees of freedom, i.e. one oscillator equation describing one eigenmode of the transmission line [9]. A distributed system actually has many standing waves and the ideal transmission line has resonances at integer multiples of the fundamental resonant frequency, $n f_{0}$. Deviations from this ideal behaviour occur due to finite coupling to input and output ports, as well as the frequency shift due to the linear part of the weak-link inductance. Because we observe IMPs of 15th order near the fundamental resonance, one really should examine the first 15 eigenmodes to determine if they are excited by harmonics or intermodulation products of the signal and pump. If so, we expect additional loss of signal power, diminishing the performance of the amplifier. Since the frequencies of these higher eigenmodes are out of the band of our HEMT amplifier, we are unable to measure their response and typically they are not measured in other experiments with cavity parametric amplifiers. Note however, that we do not expect excitation near even multiples of $f_{0}$ because $g_{\text {even }}=0$ for the nonlinear oscillator considered here. The excitation of higher eigenmodes by harmonics and intermodulation might be avoided by designing lumped-element resonators with a more complicated mode structure [27], but it is a potential problem in designs which use separate transmission line resonators to store the signal and idler [12]. Filtering response at higher harmonics and higher order intermodulation products is generally more difficult to achieve when the quality factor of the eigenmodes is decreased to achieve larger bandwidth of the amplifier.

In conclusion, we have realized phase-insensitive amplification of a microwave signal at 7.6 GHz using a nonlinear superconducting resonator pumped close to bifurcation. A peak gain of $28 \mathrm{~dB}$ was realized, but it was strongly frequency-dependent in a narrow band with a $3 \mathrm{~dB}$ bandwidth of only $1 \mathrm{MHz}$. We used a calibrated resistive thermometer to calibrate the microwave noise measurement and with the following HEMT amplifier we measured the standard quantum limit on noise, $T_{Q}=\frac{h f}{2 k_{\mathrm{B}}}$, where the noise temperature of the HEMT amplifier was only $T_{N}^{\mathrm{HEMT}}=2.6 \mathrm{~K}$, corresponding to $15 T_{Q}$. When operated as an amplifier the nonlinear resonator achieved $14 \mathrm{~dB}$ of signal gain in a $10 \mathrm{kHz}$ bandwidth with noise temperature $T_{N}^{\mathrm{NLO}}=0.44 \mathrm{~K}$, corresponding to $2.5 T_{Q}$. We were not able to demonstrate squeezing of vacuum fluctuations by examining correlations between the signal and 3rd order idler. Correlations involving higher order idlers were not examined. 


\section{Authors' contributions}

EAT and DBH conceived the experiment. EAT and AE performed the experiments and analysis. All authors took part in preparation of the manuscript.

\section{Author details}

${ }^{1}$ Intermodulation Products AB, Solna, Sweden. ${ }^{2}$ Nanostructure Physics, Royal Institute of Technology (KTH), Stockholm, Sweden.

\section{Acknowledgements}

We acknowledge stimulating discussions with Steve Girvin, Jack Lidmar, Hans Hansson, Per Delisng and Chris Wilson. This work was funded by the EU project SCOPE, FET-Open grant number 218783, the Swedish Research Council VR, the Kunt and Allice Wallenberg Foundation and the Olle Engkvist Foundation.

Received: 31 August 2013 Accepted: 6 January 2014 Published: 7 March 2014

\section{References}

1. Gao J, Vissers MR, Sandberg MO, da Silva FCS, Nam SW, Pappas DP, Wisbey DS, Langman EC, Meeker SR, Mazin BA, Leduc HG, Zmuidzinas J, Irwin KD: Appl Phys Lett 2012, 101(14):142602.

2. Houck AA, Schuster DI, Gambetta JM, Schreier JA, Johnson BR, Chow JM, Frunzio L, Majer J, Devoret MH, Girvin SM, Schoelkopf RJ: Nature 2007, 449(7160):328.

3. Hoi IC, Wilson CM, Johansson G, Palomaki T, Peropadre B, Delsing P: Phys Rev Lett 2011, 107:073601.

4. Boissonneault M, Doherty AC, Ong FR, Bertet P, Vion D, Esteve D, Blais A: Phys Rev A 2012, 85:022305.

5. Paik H, Schuster DI, Bishop LS, Kirchmair G, Catelani G, Sears AP, Johnson BR, Reagor MJ, Frunzio L, Glazman LI, Girvin SM, Devoret MH, Schoelkopf RJ: Phys Rev Lett 2011, 107:240501.

6. Clerk AA, Devoret MH, Girvin SM, Marquardt F, Schoelkopf RJ: Rev Mod Phys 2010, 82:1155.

7. Caves CM: Phys Rev D 1982, 26(8):1817.

8. Mallet F, Castellanos-Beltran MA, Ku HS, Glancy S, Knill E, Irwin KD, Hilton GC, Vale LR, Lehnert KW: Phys Rev Lett 2011, 106:220502.

9. Yurke B, Buks E: J Lightwave Technol 2006, 24(12):5054.

10. Tholén EA, Ergül A, Doherty EM, Weber FM, Grégis F, Haviland DB: Appl Phys Lett 2007, 90(25):253509.

11. Abdo B, Suchoi O, Segev E, Shtempluck O, Blencowe M, Buks E: Europhys Lett 2009, 85(6):68001.

12. Bergeal N, Schackert F, Metcalfe M, Vijay R, Manucharyan VE, Frunzio L, Prober DE, Schoelkopf RJ, Girvin SM, Devoret MH: Nature 2010, 465(7294):64

13. Abdo B, Kamal A, Devoret M: Phys Rev B 2013, 87:014508.

14. Yaakobi O, Friedland L, Macklin C, Siddiqi I: Phys Rev B 2013, 87:144301.

15. Wustmann W, Shumeiko V: Phys Rev B 2013, 87:184501.

16. Franken PA, Hill AE, Peters CW, Weinreich G: Phys Rev Lett 1961, 7:118.

17. Kamal A, Clarke J, Devoret MH: Phys Rev B 2012, 86:144510,

18. Stannigel K: Parametric amplification in superconducting resonators. Master's thesis. KTH; 2007

19. Tholén EA, Ergül A, Stannigel K, Hutter C, Haviland DB: Phys Scr 2009, T137:014019.

20. Tholén E: Intermodulation in microresonators: for microwave amplification and nanoscale surface analysis. Ph.D. thesis. KTH, Nanostructure Physics; 2009. http://kth.diva-portal.org/smash/get/diva2:277877/FULLTEXT01.pdf.

21. STAR Cryoelectronics, Santa Fe, New Mexico. http://www.starcryo.com/.

22. Low Noise Factory, Göteborg, Sweden. http://www.lownoisefactory.com/.

23. Castellanos-Beltran MA, Irwin KD, Hilton GC, Vale LR, Lehnert KW: Nat Phys 2008, 4(12):929.

24. Mariantoni M, Menzel EP, Deppe F, Araque Caballero MA, Baust A, Niemczyk T, Hoffmann E, Solano E, Marx A, Gross R: Phys Rev Lett 2010, 105:133601

25. Platz D, Forchheimer D, Tholén EA, Haviland DB: Nanotechnology 2012, 23(26):265705

26. Tholén EA, Platz D, Forchheimer D, Tholén MO, Hutter C, Haviland DB: Rev Sci Instrum 2011, 82:026109. arXiv:1008.2722v1.

27. Levenson-Falk EM, Vijay R, Siddiqi I: Appl Phys Lett 2011, 98:123115.

doi:10.1140/epjqt5

Cite this article as: Tholén et al.: Gain, noise and intermodulation in a nonlinear superconducting resonator. EPJ

Quantum Technology 2014 1:5. 